\title{
410 - Dementia-related Psychosis Symptoms and Impact from the Patient and Care Partner (Caregiver) Perspective: an Observational, Prospective Study to Describe the Patient Experience
}

\author{
Authors: Teresa Brandt ${ }^{1}$, Theresa Frangiosa ${ }^{2}$, Virginia Biggar ${ }^{2}$, Angela Taylor ${ }^{3}$, Bill Keller ${ }^{1}$, Vic Abler ${ }^{1}$ \\ ${ }^{1}$ ACADIA Pharmaceuticals Inc., San Diego, CA, USA \\ ${ }^{2}$ UsAgainstAlzheimer's (UsAA), Washington DC, USA \\ ${ }^{3}$ Lewy Body Dementia Association, Lilburn, GA, USA
}

Background: This study aimed to describe the person-centered experience of symptoms and impact of dementia-related psychosis (DRP) from a patient and care partner (CP) perspective.

Methods: This observational, non-interventional, prospective study collected patient experience data to understand the impact of DRP in persons with various dementia subtypes. Qualitative interviews were conducted with persons or their $\mathrm{CP}$ who have a clinical diagnosis of all-cause dementia with psychotic symptoms, recruited through physician referrals. The quantitative online survey was completed by persons with self-reported DRP or their CP recruited by advocacy groups: UsAgainstAlzheimer's and the Lewy Body Dementia Association. CP burden was beyond the scope of this study.

Results: Sixteen individuals ( 1 patient, $15 \mathrm{CP}$ ) participated in the qualitative interview. The most commonly reported symptoms of DRP were visual hallucinations (88\%), auditory hallucinations (69\%), and persecutory delusions (56\%). Participants described an impact of DRP on activities of daily living (75\%), sleep (63\%), family life (56\%), and safety concerns (56\%).

The quantitative portion was completed by 212 participants ( 26 patients, $186 \mathrm{CP}$ ). DRP symptoms most frequently reported by patients were visual hallucinations (89\%), auditory hallucinations (54\%), and distortion of senses (54\%); those reported by CP were paranoid delusions (76\%), visual hallucinations (75\%), and lack of trust for loved ones (52\%). For patients with recent visual hallucinations, $61 \%$ of patients and $70 \%$ of CP reported experiencing this symptom on a weekly basis. When asked to rank the impact on the patient's life, patients reported that visual hallucinations were the most impactful symptom whereas $\mathrm{CP}$ reported paranoid delusions/false beliefs as most impactful, followed by visual hallucinations. Difficulty differentiating what is real from what is not real, increased anxiety, and effects on personal relationships were the most common types of impacts reported by both patients and CP. Most patients (>40\%) were not receiving medications for DRP.

Conclusions: Persons with DRP experience hallucinations and delusions that have a substantial impact on their lives. Here, patient experience data demonstrate that there is a need for treatments to reduce the symptoms and impacts of DRP.

Study Sponsored By: ACADIA Pharmaceuticals Inc.

\section{Disclosures}

TF is a consultant with Frangiosa \& Associates, LLC.

$V B$ and $A T$ have no relevant financial relationships to disclose.

TB, BK, and VA are employees of and may hold stock and/or stock options with ACADIA Pharmaceuticals Inc. 